Pacific Journal of Mathematics

ON THE ALGEBRAIC PART OF AN ALTERNATING LINK 


\title{
ON THE ALGEBRAIC PART OF AN ALTERNATING LINK
}

\author{
Morwen B. Thistlethwaite
}

A simple method is given for determining the algebraic part of an alternating link. It is proved that the only alternating diagrams of elementary links are the "obvious" ones.

1. Introduction. For each classical link $L$ in the 3-sphere, whose complement is irreducible and geometrically atoroidal, it is explained in [B-S] how the pair $\left(S^{3}, L\right)$ admits a decomposition, unique up to isotopy, into an algebraic part $(A, L \cap A)$ and a non-algebraic part $(N, L \cap N)$. The algebraic part $A$ is constituted, in a manner explained in $\S 2$ below, from so-called "elementary tangles" in $\left(S^{3}, L\right)$; $N$ is just the closure in $S^{3}$ of $S^{3}-A$. As proved in [B-S], if the complement of $L$ is not a Seifert manifold, the submanifold $A$ of $S^{3}$ may be characterized in more general terms as follows: (i) $\partial A$ meets $L$ transversely; (ii) if $\pi: \widetilde{X} \rightarrow S^{3}$ is the 2-fold covering of $S^{3}$ branched along $L$, we may choose the characteristic variety $V$ of $\widetilde{X}$ so that $\pi^{-1}(A)$ is precisely the union of the closed-up Seifert fibered components of $\widetilde{X}-V$. If the complement of $L$ is a Seifert manifold, $A$ is either empty or equal to $S^{3}$ (see [B-S] for full details).

Since the characteristic variety $V$ of $\widetilde{X}$ consists of incompressible tori and $S^{3}-L$ is atoroidal, each component of $\partial A=\partial N$ is a 2sphere meeting the link $L$ transversely in four points. The link $L$ is said to be algebraic, or arborescent, if the algebraic part is the whole of $S^{3}$.

The main purpose of this article is to describe and justify a simple method for determining the algebraic part of $\left(S^{3}, L\right)$ in the case where the link $L$ is presented as an alternating link. It is proved in [M1] that if $L$ admits a connected, alternating diagram which is prime (in the 2-dimensional sense), then $S^{3}-L$ is irreducible and geometrically atoroidal. Therefore, in this paper, we shall be concerned with precisely those links admitting connected, prime, alternating diagrams. The method for finding the algebraic part of $\left(S^{3}, L\right)$ is based largely on Menasco's "visibility" results for Conway spheres [M1], and has already been investigated by F. Bonahon and L. Siebenmann. 
Indeed, Bonahon and Siebenmann have previously observed that the method certainly achieves the desired objective, except possibly in the awkward case where the pair $\left(S^{3}, L\right)$ contains no Conway sphere. It is explained in $\S 4$ below how the Kauffman 2-variable polynomial may be used to deal with this awkward case; in fact, the needed result is a special case of Theorem 2 of [T2], but we feel it appropriate to give in this paper an independent and more natural proof of this result. The paper is concluded with some observations on the status of the Tait flyping conjecture, and a curious classification result for Conway spheres in alternating links. I am indebted to Francis Bonahon for illuminating discussions.

2. Background. There now follows a rapid review of the underlying concepts. The definitions of this section are gleaned more or less directly from [B-S]; a minor variance of convention is that the term "Conway sphere" refers here to a pairwise essential 2-sphere meeting the link or tangle transversely in four points, and that "Conway disks" are likewise assumed to be pairwise essential.

Definition 2.1. A tangle is a pair $(X, T)$, where $X$ is a (closedup) punctured 3-sphere with at least one boundary component, and $T$ is a proper 1-submanifold of $X$, such that for each (2-sphere) component $F$ of $\partial X, \partial T \cap F$ consists of four points. Two tangles are equivalent if they are homeomorphic as pairs.

This definition generalizes the notion of tangle given, say, in [L]. In keeping with our basic assumption in this paper that links are prime, we shall assume that $X$ does not admit a 3-ball in its interior meeting $T$ in a single knotted arc.

A tangle $(X, T)$ with $n$ boundary components admits a regular projection onto a 2-sphere (extended plane) with $n$ open disks removed, $\Delta$ say (we shall refer to $\Delta$ loosely as an " $n$-punctured 2sphere"); as with links, a tangle diagram is obtained from the projection of $T$ in $\Delta$ by introducing an overcrossing-undercrossing structure (e.g. Fig. 1(i)). The type of the tangle can be recovered by a suitable small vertical perturbation near each crossing of the diagram; the particular tangle representative thus obtained from a diagram $D$ will be denoted $l(D)$. Of course, we take the boundary components of $X$ to be 2-spheres for which the boundary components of $\Delta$ are equatorial curves. We shall say that the diagram $D$ represents $(X, T)$ if the tangles $(X, l(D))$ and $(X, T)$ are equivalent, and that tangle diagrams $D_{1}, D_{2}$ are equivalent if the associated tangles $\left(X, l\left(D_{1}\right)\right)$, 


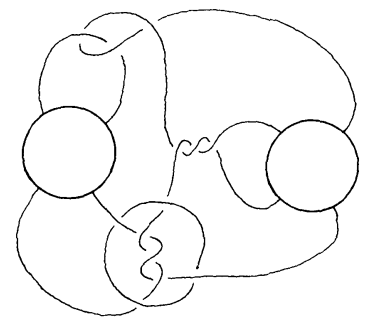

(i)

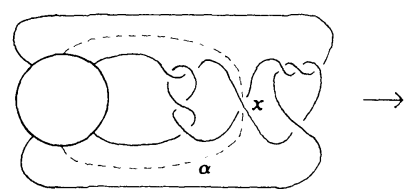

(ii)

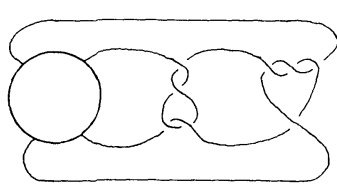

(iii)

\section{FIGURE 1}

$\left(X, l\left(D_{2}\right)\right)$ are equivalent. We shall use the notation $l(D)$ described in the previous paragraph for link diagrams also.

Let $D$ be a tangle diagram on a 2-sphere with $n$ open disks removed, denoted $\Delta$ as above. We shall assume throughout this paper that there is not any simple closed curve in $\Delta$ meeting $D$ in just one point (this point would be a "nugatory" crossing); however, we shall need to consider inessential crossings of $D$, defined as follows.

Definition 2.2. A crossing-point $x$ of a tangle diagram $D$ on an $n$-punctured 2-sphere $\Delta$ is inessential if there exists an arc $\alpha \subset \Delta$, with $\alpha \cap D=x$, such that both ends of $\alpha$ lie on the same boundary component of $\Delta$ and $\alpha$ separates the four arcs of $D$ incident upon $x$ into two pairs. A tangle diagram is reduced if it contains no inessential crossings.

An example of an inessential crossing is illustrated in Fig. 1(ii). Clearly, any inessential crossing may be removed by twisting in such a way that part of the diagram is inverted, without disturbing the type of the tangle (Fig. 1(iii)). If an inessential crossing is removed in this way from an alternating diagram, the resulting diagram is also alternating, with one less crossing.

Definition 2.3. A trivial tangle with one boundary component (resp. with two boundary components) is a tangle homeomorphic to that illustrated in Fig. 2(i) (resp. Fig. 2(ii)). Thus a trivial tangle with two boundary components is homeomorphic to $\left(S^{2}, 4\right.$ points $) \times I$. A hollow elementary tangle is a tangle homeomorphic to the tangle with three boundary components illustrated in Fig. 2(iii). An elementary tangle is a tangle obtained from a hollow elementary tangle $(X, T)$ by glueing a trivial tangle of one boundary component to each of 0,1 or 2 boundary components of $(X, T)$. If all three boundary components of a hollow elementary tangle are plugged in this manner with trivial tangles of one boundary component, the resulting object is called an 


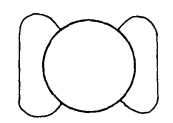

(i)

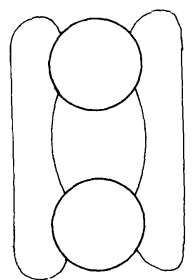

(ii)

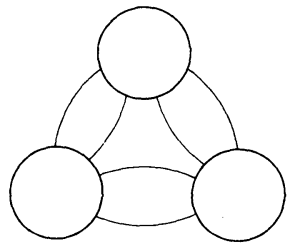

(iii)

FIGURE 2

elementary link pair. See Fig. 6 for examples of elementary tangles, and an example of an elementary link.

As is well known, given a suitable coordinate system, the attaching map of a tangle of one boundary component is determined up to isotopy by an element of $\mathbb{Q} \cup\{\infty\}$; a trivial tangle together with this glueing information is called a rational tangle. Note that an elementary link, as defined above, is merely a Montesinos link with at most three rational substituents.

Definition 2.4. A Conway sphere in a tangle $(X, T)$ is a 2-sphere $F$ in $\operatorname{int}(X)$, meeting $T$ transversely in four points, such that the closure of neither component of $(X, T)-(F, F \cap T)$ is a trivial tangle with one or two boundary components.

In the presence of the assumption in this paper that $(X, T)$ does not contain in its interior a 3-ball meeting $T$ in a single knotted arc, a 2-sphere in $\operatorname{int}(X)$ meeting $T$ transversely in four points bounds a trivial tangle with one boundary component in $(X, T)$ if and only if it admits a pairwise compression disk; also, the closure of some component of $(X, T)-(F, F \cap T)$ is a trivial tangle with two boundary components if and only if $F$ is pairwise parallel to the boundary of $X$. For definitions of "pairwise essential", "pairwise parallel", see [B-S]. A Conway sphere in a link pair $\left(S^{3}, L\right)$ is defined likewise; we merely omit the condition regarding trivial tangles with two boundary components. We shall say that two Conway spheres are parallel if they are parallel in the pairwise sense, i.e. if they bound a trivial tangle with two boundary components.

Definition 2.5. A Conway disk in a tangle pair $(X, T)$ is a disk $D$ properly embedded in $X, \partial D$ being contained in some component $W$ say of $\partial X$, with the following properties: $\partial D$ does not meet $T$, $\operatorname{int}(D)$ meets $T$ in two points, each component of $W-\partial D$ meets $T$ in two points, and $D$ is not pairwise parallel to $W$ (i.e. there does not 
exist an isotopy, fixing $\partial D$, of $D$ into $W$, through disks all meeting $T$ in two points).

The preimage in the twofold cover $\tilde{X}$ of $X$ branched along $T$ of a Conway sphere is an essential torus, and the preimage in $\widetilde{X}$ of a Conway disk is an essential annulus, both of whose boundary components lie on the same boundary component of $\widetilde{X}$.

Note that elementary tangles and links do not admit Conway spheres: the double branched cover of such a tangle or link cannot contain an essential torus, as it admits a Seifert fibration over a planar surface, where the sum of the numbers of boundary components and singular fibers is at most three. Also, we can see immediately by looking at double branched covers that a trivial tangle with one or two boundary components cannot admit a Conway disk: the double branched cover of a trivial tangle with one boundary component is a solid torus, and the double branched cover of a trivial tangle with two boundary components is a product of a torus with an interval. However, each elementary tangle $(X, T)$, which is not a trivial tangle with one or two boundary components, admits a Conway disk (illustrated as a dotted line in Fig. 6(i), (ii)), visibly separating the tangle into two trivial tangles, $\left(X_{1}, T_{1}\right),\left(X_{2}, T_{2}\right)$ say; this disk cannot be pairwise parallel to the boundary of $(X, T)$, for otherwise $(X, T)$ would be equivalent to one of the $\left(X_{i}, T_{i}\right)$ and would thus be trivial. Conversely, it is easy to see that any tangle manufactured by glueing together two trivial tangles along a Conway disk has a diagram of the general form of Fig. 6(i) or (ii); hence such a tangle is elementary.

Next, we make the observation that if $D$ is a Conway disk in a tangle $(X, T)$ for which some closed-up component $(Y, U)$ of $(X, T)-$ $(D, D \cap T)$ is non-trivial, then $(X, T)$ contains a Conway sphere, namely any 2 -sphere in $\operatorname{int}(Y)$ pairwise parallel to $\partial Y$. Because of this, the only Conway disks of interest to us in alternating diagrams will be those occurring in elementary tangles.

From the above discussion, we have the following characterizations of non-trivial elementary tangles.

Proposition 2.6. The following statements are equivalent:

(i) $(X, T)$ is a non-trivial elementary tangle;

(ii) $(X, T)$ may be cut along a Conway disk into two trivial tangles;

(iii) $(X, T)$ admits a Conway disk, but does not admit a Conway sphere. 
DEFINITION 2.7. A link or tangle pair is algebraic if it is elementary, or if it can be cut along a collection of Conway spheres into elementary tangles.

Of course, the double branched cover of an algebraic link or tangle pair is a graph-manifold [B-S]; the decomposition just described corresponds to cutting the graph-manifold along incompressible tori into pieces, each of which is homeomorphic to $\left(S^{2}-3\right.$ open disks $) \times S^{1}$ plugged with 0,1 or 2 solid tori. The double branched cover of an elementary link is merely $\left(S^{2}-3\right.$ open disks $) \times S^{1}$ plugged with three solid tori.

DEFINITION 2.8. Let $F_{1}, \ldots, F_{n}$ be a maximal, finite collection of pairwise disjoint and non-parallel Conway spheres in $\left(S^{3}, L\right)$ (such a collection exists by Haken finiteness: see [B-S], §4.2). Then the algebraic part of $\left(S^{3}, L\right)$ is the union of those closed-up components of $S^{3}-\bigcup_{i} F_{i}$ which are elementary.

As already mentioned, in determining the algebraic part of a link pair $\left(S^{3}, L\right)$, it is necessary to consider separately the cases when $\left(S^{3}, L\right)$ admits a Conway sphere, and when it does not. In the first of these cases, clearly the ability to recognize Conway spheres and elementary tangles is pertinent; this is dealt with in the next section.

3. Recognizing Conway spheres and elementary tangles in alternating diagrams. First, let us recall Menasco's “visibility" result concerning Conway spheres.

Let $L$ be a link which is $l(D)$ for some alternating diagram $D$. Then it is shown in [M1], by elegant and elementary means, that each Conway sphere for $L$ is parallel to one whose intersection with the 2-sphere $S_{+}$of [M1] (i.e. the projection 2-sphere perturbed slightly so as to contain the overpasses) consists of either one or two circles, as in Fig. 3(i, ii). The method of proof is equally valid for tangle diagrams. Let us say that a Conway sphere is visible for a diagram $D$ if it is parallel to one conforming to Fig. 3(i), and let us say that it is hidden otherwise. We may assume that each shaded disk in Fig. 3(ii) represents a portion of diagram with at least one crossing; otherwise the Conway sphere will be parallel to one conforming to Fig. 3(i) For example, the alternating diagram obtained by substituting a single crossing for each of these shaded disks is the usual 6-crossing diagram of the Borromean rings.

Let $(X, l(D))$ be a tangle corresponding to an alternating diagram $D$ in a punctured 2-sphere $\Delta$, and let $F$ be a Conway sphere in 


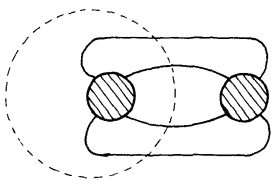

(i)

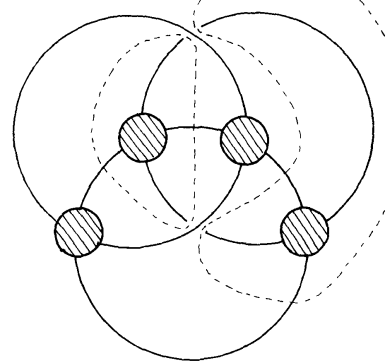

(ii)

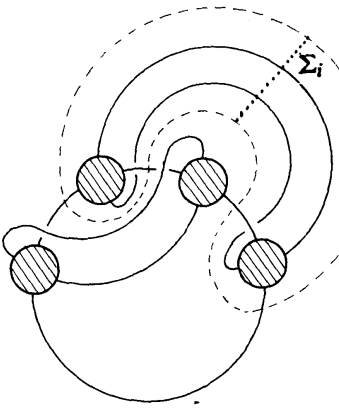

(iii)

\section{FIGURE 3}

$X$. If $F$ is visible, then cutting $X$ along $F$ clearly results in two tangles $l\left(D_{1}\right), l\left(D_{2}\right)$, where $D_{1}, D_{2}$ are simply the alternating tangle diagrams obtained by cutting $\Delta$ along $F \cap \Delta$. On the other hand, if $F$ is hidden, there is an isotopy of $X$ which transforms $D$ to a non-alternating diagram with two extra crossings, in which the image of $F$ under this isotopy is now visible (Fig. 3(iii)). The two tangles $\left(X_{1}, T_{2}\right),\left(X_{2}, T_{2}\right)$ obtained by cutting $X$ along $F$ might not admit alternating diagrams, but we can cut each $\left(X_{i}, T_{i}\right)$ along a disk $\Sigma_{i}$ meeting $T_{i}$ in two points, indicated in Fig. 3(iii) by a dotted line, into two (possibly trivial) tangles which visibly admit alternating diagrams. We shall see, in Corollary 3.4 below, that this disk $\Sigma_{i}$ is indeed a Conway disk for $\left(X_{i}, T_{i}\right)$.

In the case of the Borromean rings, Fig. 3(iii) exhibits a decomposition of this (3-sphere, link)-pair into two elementary tangles, each presented as a diagram with four crossings. Therefore the "Borromean rings" is an algebraic link according to Definition 2.7; it is the simplest example of an alternating link which is algebraic, but which does not admit an alternating, "algebraic" diagram in the sense of [C] (recall from [C] that a diagram is algebraic if it has Conway basic polyhedron $\left.1^{*}\right)$.

At this point, the reader might wonder how we can tell whether our 2-sphere $F$, meeting the link or tangle in four points, really is a Conway sphere (the same question arises for purported Conway disks). According to Definition 2.4, this amounts to deciding whether $F$ abuts a non-trivial tangle on both sides. This question has been dealt with by geometric means in Menasco's paper [M2], but here we provide an alternative approach, using properties of the Kauffman polynomial proved in [T3]. The reader is referred to [T3] for the definition of adequate link diagram. 


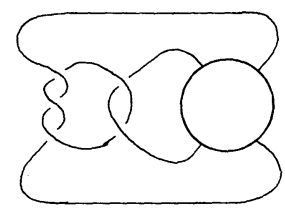

$(X, T)$

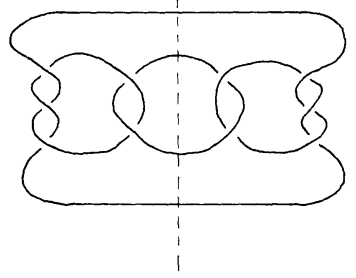

Double of $(X, T)$

\section{Figure 4}

The next theorem provides a stronger result than is needed for the applications; it may be of independent interest.

THEOREM 3.1. Let $D$ be a reduced alternating diagram, with $n$ crossings, of a tangle $(X, T)$ with one boundary component. Then no diagram representing that tangle has fewer than $n$ crossings.

Proof. We can form the "double" of any diagram of $T$, as illustrated in Fig. 4, to obtain a diagram of a (3-sphere, link)-pair which is a pure double of $(X, T)$. The type of this link is evidently independent of the particular diagram of $T$. If we double a reduced alternating tangle diagram in this way, a (non-alternating) adequate link diagram is produced, which has minimal crossing-number for the link it represents by Corollary 3.4 of [T3].

REMARK. Clearly the proof of Theorem 3.1 applies equally to any tangle diagram whose double is an adequate link diagram.

COROLlary 3.2. A reduced alternating diagram with at least one crossing cannot represent a trivial tangle with one or two boundary components.

Proof. Since trivial tangles admit diagrams with no crossing, the result for tangles with one boundary component follows directly from Theorem 3.1. Now let $(X, T)$ be a trivial tangle with two boundary components. Let $\left(X_{1}, T_{1}\right)$ be the result of glueing some tangle $(Y, U)$ to one of the boundary components of $(X, T)$. Then, sinee $(X, T)$ is equivalent to $\left(S^{2}, 4\right.$ points $) \times I,(Y, U)$ is equivalent to $\left(X_{1}, T_{1}\right)$. Suppose that $D$ is a reduced, alternating diagram with $n$ crossings of $(X, T)$, in a twice-punctured 2-sphere $\Delta$. Then we can adjoin a diagram with no crossing of a trivial tangle with one boundary component to one of the boundary components of $(\Delta, D)$, to obtain 
a reduced diagram $D_{1}$ with $n$ crossings. According to the above argument, $D_{1}$ represents a trivial tangle with one boundary component; hence by Theorem $3.1 n=0$.

It follows in particular that any alternating diagram of a trivial tangle with one boundary component is a 4-string plait diagram, and that any alternating diagram of a trivial tangle with two boundary components is a diagram of a 4-string geometric braid in $S^{2} \times I$.

COROLlARY 3.3. Let $D$ be a reduced alternating diagram of a tangle (or link) $(X, l(D))$, let $F$ be a hidden Conway sphere for $(X, l(D))$, and let $\left(X_{1}, T_{1}\right),\left(X_{2}, T_{2}\right)$ be the tangles obtained by cutting $(X, l(D))$ along $F$. Then

(i) Each tangle $\left(X_{1}, T_{1}\right),\left(X_{2}, T_{2}\right)$ is non-trivial; moreover, if some $\left(X_{i}, T_{i}\right) \quad(i=1,2)$ has one boundary component, then the diagram of $\left(X_{i}, T_{i}\right)$ illustrated in Fig. 3(iii) has minimal number of crossings for diagrams representing $\left(X_{i}, T_{i}\right)$.

(ii) The disk $\Sigma_{i}$ in $\left(X_{i}, T_{i}\right)$ indicated by a dotted line in Fig. 3(iii) is a Conway disk for $\left(X_{i}, T_{i}\right)$.

Proof. (i) For $i=1,2$ let $D_{i}$ be the (non-alternating) diagram of $\left(X_{i}, T_{i}\right)$, obtained as in Fig. 3(iii). If $\left(X_{i}, T_{i}\right)$ has one boundary component, then the double of $D_{i}$ is an adequate link diagram; hence, by the remark following Theorem 3.1, $D_{i}$ has minimal crossing-number for $\left(X_{i}, T_{i}\right)$, and in particular that tangle cannot be trivial. On the other hand, if $\left(X_{i}, T_{i}\right)$ has two boundary components, the method of proof of Corollary 3.2 shows that $\left(X_{i}, T_{i}\right)$ is non-trivial.

(ii) Suppose $\Sigma_{i}$ were pairwise parallel to the boundary of $\left(X_{i}, T_{i}\right)$. Then there would be a 2 -sphere $G$, made up from $\Sigma_{i}$ with a small open collar of its boundary removed, together with a disk close to and parallel to one of the components of $\partial X_{i}-\partial \Sigma_{i}$, such that $G$ and $\partial X_{i}$ bounded a trivial tangle of two components (Fig. 3(iii)). But the tangle bounded by $G$ and $\partial X_{i}$ has a reduced, alternating diagram with at least two crossings, so this tangle cannot be trivial.

From Corollary 3.2, one may determine whether an alternating diagram of a tangle with one or two boundary components represents a trivial tangle by repeatedly removing inessential crossings until a reduced alternating diagram is obtained. Then the tangle is trivial if and only if that reduced diagram has no crossings. 
Corollaries 3.3 and 3.4 enable us to deal effectively with hidden Conway spheres. Using the notation of Corollary 3.3, one looks at the (alternating) subtangles into which $\left(X_{i}, T_{i}\right)$ is decomposed by cutting along the Conway disk $\Sigma_{i}$. If both these subtangles are seen to be trivial, then $\left(X_{i}, T_{i}\right)$ is elementary by Proposition 2.6(ii); otherwise, $\left(X_{i}, T_{i}\right)$ contains a visible Conway sphere, i.e. one such as $G$ in Fig. 3(iii), and cutting along this sphere decomposes $\left(X_{i}, T_{i}\right)$ into two alternating, non-trivial tangles.

Let us pause for a moment, to see where we stand in our quest for a determination of the algebraic part of $\left(S^{3}, L\right)$, in the case where $\left(S^{3}, L\right)$ admits a Conway sphere. Given an alternating link or tangle Diagram $D$, we have described a method for detecting 2-spheres meeting the link or tangle $l(D)$ in four points, and a method for determining whether that 2-sphere is pairwise essential. We have also dealt fully with the consequences of cutting along a hidden Conway sphere. Therefore, apart from the matter of recognizing elementary tangles resulting from cuts along visible Conway spheres, the procedure is now clear. One cuts $\left(S^{3}, L\right)$ along a Conway sphere, then cuts the resulting pieces likewise, and so on, continuing the process until one has obtained a collection of non-trivial tangle pieces of $\left(S^{3}, L\right)$, none of which admits a Conway sphere. All that remains is to be able to determine which pieces of this collection are elementary; from Proposition 2.6, this is equivalent to determining which pieces admit Conway disks.

Now, Menasco's analysis of Conway spheres for alternating diagrams may easily be adapted to deal with Conway disks. It turns out that, as with spheres, there are "visible" and "hidden" Conway disks (Fig. 5). However, since a hidden Conway disk always abuts a nontrivial tangle, we shall not need to consider these: observe that in Fig. 5(iii) one of the tangles obtained by cutting along the Conway disk has a diagram of the same sort as that occurring in Fig. 3(iii). The upshot is that Conway disks in reduced alternating diagrams of elementary tangles are always visible; moreover, since any alternating diagram of a trivial tangle with one boundary component is a 4-string plait diagram, any alternating diagram of a non-trivial elementary tangle consists of 4-string plaits and boundary components set out in thẹ obvious fashion (Fig. 6). Therefore elementary tangles are recognizable from their alternating diagrams, and, in the case where $\left(S^{3}, L\right)$ admits a Conway sphere, the process of determining the algebraic part of $\left(S^{3}, L\right)$ is now complete. 


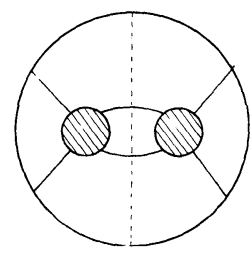

(i)

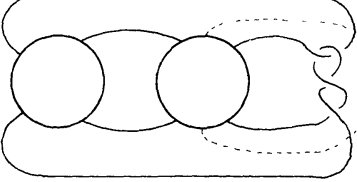

Typical elementary tangles

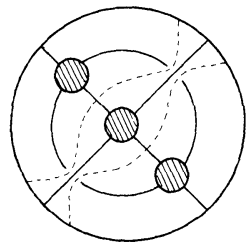

(ii)

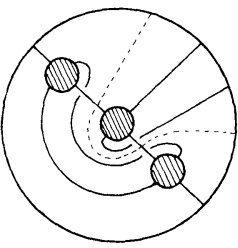

(iii)

FIGURE 5

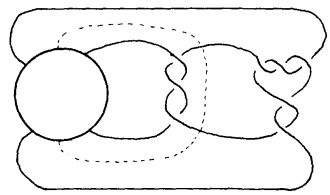

FIGURE 6

4. The case where $\left(S^{3}, L\right)$ does not admit a Conway sphere. We shall assume throughout this section that $L=l(D)$ for some reduced alternating link diagram $D$, and that there is no Conway sphere in $\left(S^{3}, L\right)$. From Definition 2.8, we see at once that either $L$ is an elementary link, or else the algebraic part of $\left(S^{3}, L\right)$ is empty. The aim of this section is to show that, as with elementary tangles, the only alternating diagrams of elementary links are the "obvious" ones.

Let us recall that an elementary link is merely a Montesinos link with at most three rational substituents. In [L-T], the notion of a reduced Montesinos diagram of a Montesinos link is described, and it is proved that a Montesinos link cannot be projected with fewer crossings than in such a diagram. It then follows from Theorems 1 and 2 of [T1] that if $L$ admits a non-alternating reduced Montesinos diagram, then $L$ cannot admit any alternating diagram; therefore we can immediately discount links whose reduced Montesinos presentations are non-alternating.

Now suppose $L$ is an alternating, elementary link. Either $L$ is 2-bridged and admits a standard 4-string plait diagram, or $L$ admits an alternating, reduced Montesinos diagram with three substituent rational tangles, of the type illustrated in Fig. 6(iii). Indeed, if $D$ is any alternating, algebraic diagram of $L$, i.e. an alternating diagram whose Conway basic polyhedron is $1^{*}[\mathbf{C}]$, then from the absence of Conway spheres in $\left(S^{3}, L\right)$ it is easily seen that $D$ must be either a 4-string plait diagram or a reduced Montesinos diagram. However, we must still consider the possibility that $L$ might admit an 
alternating diagram whose Conway basic polyhedron is other than $1^{*}$. This possibility is duly excluded by the following theorem.

THEOREM 4.1. If a link $L$ admits an alternating diagram which is algebraic, then every alternating diagram of $L$ is algebraic.

A longer proof of a slightly more general theorem is set forth in [T2]; there it is proved that the basic polyhedra $1^{*}, 6^{*}, 8^{*}$ are all "characteristic" for reduced alternating diagrams.

As in [T2], we shall use the integer invariant $\kappa(D)$ of regular isotopy classes of alternating diagrams $D$ with at least two crossings, determined by the Kauffman two-variable polynomial $\Lambda_{D}(a, z)$. We recall the following facts about $\kappa$ :

(i) $\kappa(D) \geq 0$, and $\kappa(D)>0$ if and only if $D$ is reduced and prime;

(ii) $\kappa(D)$ depends only on the basic polyhedron of $D$;

(iii) if $D$ is algebraic, $\kappa(D)=1$.

Therefore, to prove Theorem 4.1, it will be sufficient to show that $\kappa(D)>1$ if $D$ is a reduced alternating diagram whose underlying projection is a basic polyhedron other than $1^{*}$.

Now let $G=G(D)$ be the connected, planar graph obtained from a black-and-white shading of $D$ by associating, in the usual fashion, a vertex of $G$ to each white region say of $D$, and an edge of $G$ to each crossing of $D$. It will be convenient to discuss the invariant $\kappa$ applied to $G(D)$, rather than to $D$. We shall use the standard notation $G_{e}^{\prime}$, $G_{e}^{\prime \prime}$ for the graphs obtained from $G$ by respectively deleting an edge $e$ of $G$, contracting the edge $e$. We shall say that an edge of $G$ links its endpoints.

Definition 4.2. A connected graph $G$ is separable if either of the following holds:

(i) $G$ has more than one edge, and at least one edge of $G$ is a loop;

(ii) $G$ may be disconnected by the removal of a single vertex, called a cutvertex of $G$, together with its incident edges.

Definition 4.3. A subgraph $H$ of a separable graph $G$ is a cut component of $G$ with respect to $v$ if it is a component of the graph obtained by deleting from $G$ some cutvertex $v$ of $G$ together with its incident edges. 
The nonnegative integer invariant $\kappa$ is characterized by the following properties:

I $\kappa(G)=0$ if and only if $G$ is separable;

II if $G$ is the graph $\sim, \kappa(G)=1$;

III if $G$ has at least three edges, and $e$ is an edge of $G$ which is neither an isthmus nor a loop, then

$$
\kappa(G)=\kappa\left(G_{e}^{\prime}\right)+\kappa\left(G_{e}^{\prime \prime}\right) .
$$

Note 4.4. The above characterization is somewhat redundant, as the implication $(\kappa(G)=0 \Rightarrow G$ is separable) follows from properties II, III. We leave this as an exercise to the interested reader.

Now, if the underlying projection of a diagram $D$ is a non-trivial basic polyhedron, then that diagram has no two-sided region. Therefore the planar graph $G(D)$ contains no vertex of valency 2, nor any pair of vertices linked by more than one edge (Fig. 7).

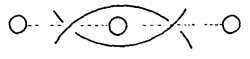

Figure 7

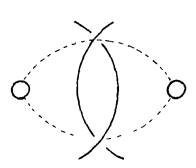

\section{7}

The proof of the Theorem is now reduced to the following.

Proposition 4.5. Let $G$ be a connected graph with at least two edges, such that $\kappa(G)=1$. Then $G$ has a vertex of valency 2 or a pair of vertices linked by more than one edge.

Proof. The conclusion certainly holds for the graph $\bigcirc$. We proceed by induction on the number of edges of $G$. Suppose the statement holds for all graphs with fewer than $n$ edges; let $G$ be a graph with $n>2$ edges, such that $\kappa(G)=1$ and $G$ fails to satisfy the conclusion of the Proposition. Since $G$ is non-separable by Property I above, $G$ contains no isthmus or loop. If $e$ is any edge of $G$, by Property III and the non-negativity of $\kappa$, precisely one of $G_{e}^{\prime}, G_{e}^{\prime \prime}$ is separable. Let us choose $e$ so that this separable graph $\left(G_{e}^{\prime}\right.$ or $G_{e}^{\prime \prime}$ ) has a cut component $H$ say with smallest possible number of edges. We shall assume henceforth that this separable graph is $G_{e}^{\prime}$; the case where the separable graph is $G_{e}^{\prime \prime}$ is dealt with by means of 
a dual argument. Therefore we assume that $G_{e}^{\prime}$ is separable with a cut component, $H$ say, with minimal number of edges for all choices of $G_{e}^{\prime}, G_{e}^{\prime \prime}$, and that $G_{e}^{\prime \prime}$ is non-separable. Since $\kappa\left(G_{e}^{\prime \prime}\right)=1$, by the inductive hypothesis $G_{e}^{\prime \prime}$ has a vertex of valency 2 or a pair of vertices linked by more than one edge. However, by hypothesis $G$ has no vertex of valency 2 , and contraction of $e$ cannot decrease vertex valencies; therefore $G_{e}^{\prime \prime}$ must have a pair of vertices linked by more than one edge. It follows that $e$ must belong to a cycle of length 3 in $G$, whose vertices are $u, v, w$ say, where $u, v$ are incident to $e$ and $w$ is the cutvertex of $G_{e}^{\prime}$ giving rise to the minimal cut component $H$ of $G_{e}^{\prime}$ (Fig. 8). As $G$ is non-separable, the graph $K$, obtained by deleting from $G$ the vertex $w$ together with its incident edges, is connected. In this graph $K, e$ is an isthmus, so $K-e$ has two components; these components are precisely the cut components of $G_{e}^{\prime}$ with respect to $w$. Therefore the minimal cut component $H$ of $G_{e}^{\prime}$ must contain precisely one of the vertices $u, v$; let this vertex be $u$. Let $e_{1}$ be the edge of $G$ joining $u, w$. Note that $H$ must contain at least one other edge $f$ say, incident to $u$ and not incident to $w$, as $u$ cannot have valency 2 in $G$ and $u, w$ cannot be linked in $G$ by more than one edge. Let $x$ be the other end of $f$ (Fig. 8). $G_{e_{1}}^{\prime \prime}$ is separable, with cutvertex the single vertex, $w_{1}$ say, obtained from the identification of $u, w$ under the contraction of $e_{1}$. But the cut component of $G_{e_{1}}^{\prime \prime}$, corresponding to $w_{1}$ and containing the vertex $x$, has fewer edges than $H$, contradicting the minimality of $H$.

5. Epilogue. An unexpected consequence of the method of proof of Theorem 3.1 is the following.

Proposition 5.1. Let $D_{1}, D_{2}$ be alternating link diagrams such that there exists a homeomorphism $h:\left(S^{3}, l\left(D_{1}\right)\right) \rightarrow\left(S^{3}, l\left(D_{2}\right)\right)$, and let $F$ be a Conway sphere for the link $l\left(D_{1}\right)$ which is visible for $D_{1}$. Then $h(F)$ is visible for $D_{2}$.

Proof. $D_{1}, D_{2}$ have the same number of crossings by Theorem 1 of [T1]; let their common crossing-number be $n$. Let $F$ be as in the statement of the proposition. Then we may assume that the intersection of $F$ with the projection plane is a circle which divides the link diagram $D_{1}$ into two tangle diagrams $U_{1}$ and $V_{1}$ say. Let us suppose that $D_{2}$ and $h(F)$ conform to Fig. 3(ii). Then, as we have already seen in $\S 3$, we may transform the diagram $D_{2}$ to a non- 
alternating diagram $D_{2}^{\prime}$ with $(n+2)$ crossings, in which the image of $h(F)$ under this isotopy has become visible (Fig. 3(iii)). Let $h^{\prime}$ be the modified homeomorphism of $S^{3}$ which carries $l\left(D_{1}\right)$ to $l\left(D_{2}\right)$. Then the intersection of $F^{\prime}=h^{\prime}(F)$ with the projection 2-sphere is a circle which divides the diagram $D_{2}^{\prime}$ into two tangle diagrams $U_{2}, V_{2}$ such that $h^{\prime}\left(U_{1}\right)=U_{2}$ and $h^{\prime}\left(V_{1}\right)=V_{2}$. Since $D_{1}$ has fewer crossings than $D_{2}^{\prime}$, it follows that at least one of the tangle diagrams $U_{2}, V_{2}$ does not have minimal crossing-number for the tangle it represents. But, as already mentioned in the proof of Corollary 3.3, doubling either of these tangle diagrams yields an adequate diagram, thereby forcing a contradiction.

Thus there are two discernible types of Conway sphere for alternating links: the type of sphere which, informally speaking, is "always visible", and the type which is "always hidden". Bonahon states that he has a purely geometric proof (unpublished) of Proposition 5.1.

Proposition 5.1 would follow from the celebrated Tait flyping conjecture, which proclaims that, given any two alternating diagrams of a link, one can transform one diagram to the other by means of a sequence of flypes ' The Tait conjecture is unsolved in general, but in light of the results of [B-S] and the discussion of the previous section, we may make the following assertion.

Proposition 5.2. Let $L$ be a link admitting an alternating, algebraic diagram. Then any two alternating diagrams of $L$ are related via a sequence of flypes.

Sketch proof. From Theorem 4.1, every alternating diagram of $L$ is algebraic. But in [B-S] it is proved that any two algebraic diagrams of a link must be related via certain moves; in particular, it follows from their analysis that any two alternating, algebraic diagrams of a link are related via a sequence of flypes.

One may deduce in particular from Proposition 5.2 that every alternating diagram representing a 2-bridged link is "standard". There does not appear to be any purely geometric proof of this available at present.

Proposition 5.2 does not cater for those alternating diagrams which represent links which are algebraic according to Definition 2.7, yet which do not have basic polyhedron $1^{*}$ (for example the 6-crossing diagram of the Borromean rings). From Proposition 5.1, if a link 

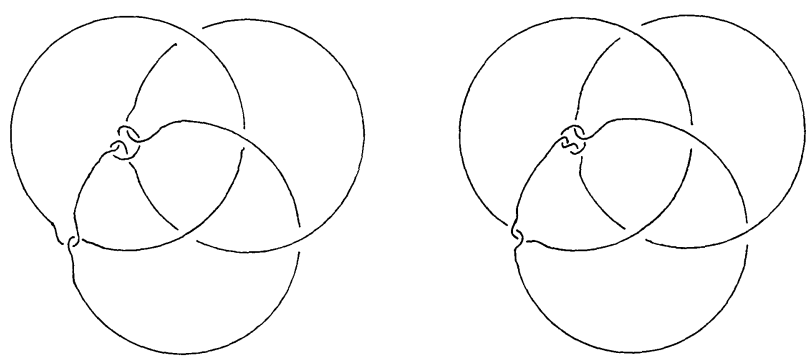

Figure 9

admits such an alternating diagram, every alternating diagram of the link must be of that form. Such a diagram can always be transformed to a non-alternating diagram which does have basic polyhedron $1^{*}$, by repeated application of the move which transforms Fig. 3(ii) to Fig. 3(iii), at which stage the machinery of [B-S] can be applied. It should be possible, by means of this approach, to show that any two alternating diagrams of an algebraic link are related via a sequence of flypes, whether or not the diagrams themselves are algebraic; however, we shall not pursue this matter here.

We close by remarking that the flyping conjecture does not hold for the more general class of adequate diagrams [T3]; Fig. 9 illustrates two adequate 11-crossing diagrams of the famous Kinoshita-Terasaka knot with trivial Alexander polynomial, each conforming to the pattern of Fig. 3(ii). These diagrams are not related via a sequence of flypes, and indeed at first sight it is not obvious that they represent the same knot type. However, their equivalence becomes fairly easy to see once they have been transformed to the pattern of Fig. 3(iii).

\section{REFERENCES}

[B-S] F. Bonahon and L. Siebenmann, Algebraic knots, preprint.

[C] J. H. Conway, An enumeration of knots and links, Computational Problems in Abstract Algebra, ed. Leech, Pergamon Press (1970), 329-358.

[L] W. B. R. Lickorish, Prime knots and tangles, Trans. Amer. Math. Soc., 267 no. 1 (1981), 321-332.

[L-T] W. B. R. Lickorish and M. B. Thistlethwaite, Some links with non-triviad polynomials and their crossing-numbers, Comment. Math. Helv., 63 (1988); 527-539.

[M1] W. Menasco, Closed incompressible surfaces in alternating knot and link complements, Topology, 23 (1984), 37-44.

[M2] _ Determining incompressibility of surfaces in alternating knot and link complements, Pacific J. Math., 117 no. 2 (1985), 353-370. 
[T1] M. B. Thistlethwaite, A spanning-tree expansion of the Jones polynomial, Topology, 26 no. 3 (1987), 297-309.

[T2] _ Kauffman's polynomial and alternating links, Topology, 27 no. 3 (1988), 311-318.

[T3] _ On the Kauffman polynomial of an adequate link, Inventiones Math., 93 (1988), 285-296.

Received August 20, 1990 and in revised form January 7, 1991.

UNIVERSITY OF TENNESSEE

KNOXVILLE, TN 37996-1300 



\section{PACIFIC JOURNAL OF MATHEMATICS EDITORS}

\author{
V. S. VARADARAJAN \\ (Managing Editor) \\ University of California \\ Los Angeles, CA 90024-1555-05 \\ Herbert Clemens \\ University of Utah \\ Salt Lake City, UT 84112 \\ THOMAS ENRIGHT \\ University of California, San Diego \\ La Jolla, CA 92093
}

Nicholas ERcolani

University of Arizona

Tucson, AZ 85721

R. FINN

Stanford University

Stanford, CA 94305

VAUGHAN F. R. JONES

University of California

Berkeley, CA 94720

STEVEN KeRCKHOFF

Stanford University

Stanford, CA 94305

\section{C. MOORE \\ University of California \\ Berkeley, CA 94720}

MARTIN SChaRLEMANN

University of California

Santa Barbara, CA 93106

HAROLd STARK

University of California, San Diego

La Jolla, CA 92093

\section{ASSOCIATE EDITORS}

\begin{tabular}{|c|c|c|c|c|}
\hline ARENS & $\begin{array}{l}\text { E. F. BECKENBACH } \\
(1906-1982)\end{array}$ & NeumanN & $\begin{array}{l}\text { F. Wolf } \\
(1904-1989)\end{array}$ & K. Yoshida \\
\hline \multicolumn{5}{|c|}{ SUPPORTING INSTITUTIONS } \\
\hline \multirow{2}{*}{\multicolumn{2}{|c|}{$\begin{array}{l}\text { IVERSITY OF ARIZONA } \\
\text { IIVERSITY OF BRITISH COLUMBIA }\end{array}$}} & UNIVERS & Y OF OREGON & \\
\hline & & UNIVERS & OF SOUTHER & IIA \\
\hline \multicolumn{2}{|c|}{ LIFORNIA INSTITUTE OF TECHNOLOGY } & STANFO & UNIVER & \\
\hline \multirow{2}{*}{\multicolumn{2}{|c|}{$\begin{array}{l}\text { IVERSITY OF CALIFORNIA } \\
\text { ONTANA STATE UNIVERSITY }\end{array}$}} & UNIVERS & OF HAWAII & \\
\hline \multirow{2}{*}{\multicolumn{2}{|c|}{$\begin{array}{l}\text { ONTANA STATE UNIVERSITY } \\
\text { IIVERSITY OF NEVADA, RENO }\end{array}$}} & UNIVERS & YF TOKYO & \\
\hline & & UNIVERS & I OF UTAH & \\
\hline \multirow{2}{*}{\multicolumn{2}{|c|}{$\begin{array}{l}\text { W MEXICO STATE UNIVERSITY } \\
\text { EGON STATE UNIVERSITY }\end{array}$}} & WASHING & N STATE UNI & SITY \\
\hline & & UNIVERS & NASI & \\
\hline
\end{tabular}

The Supporting Institutions listed above contribute to the cost of publication of this Journal, but they are not owners or publishers and have no responsibility for its content or policies.

Mathematical papers intended for publication in the Pacific Journal of Mathematics should be in typed form or offset-reproduced (not dittoed), double spaced with large margins. Please do not use built up fractions in the text of the manuscript. However, you may use them in the displayed equations. Underline Greek letters in red, German in green, and script in blue. The first paragraph must be capable of being used separately as a synopsis of the entire paper. In particular it should contain no bibliographic references. Please propose a heading for the odd numbered pages of less than 35 characters. Manuscripts, in triplicate, may be sent to any one of the editors. Please classify according to the 1991 Mathematics Subject Classification scheme which can be found in the December index volumes of Mathematical Reviews. Supply name and address of author to whom proofs should be sent. All other communications should be addressed to the managing editor, or Elaine Barth, University of California, Los Angeles, California 90024-1555-05.

There are page-charges associated with articles appearing in the Pacific Journal of Mathematics. These charges are expected to be paid by the author's University, Government Agency or Company. If the author or authors do not have access to such Institutional support these charges are waived. Single authors will receive 50 free reprints; joint authors will receive a total of 100 free reprints. Additional copies may be obtained at cost in multiples of 50 .

The Pacific Journal of Mathematics (ISSN 0030-8730) is published monthly except for July and August. Regular subscription rate: $\$ 190.00$ a year (10 issues). Special rate: $\$ 95.00$ a year to individual members of supporting institutions.

Subscriptions, orders for numbers issued in the last three calendar years, and changes of address should be sent to Pacific Journal of Mathematics, P.O. Box 969, Carmel Valley, CA 93924, U.S.A. Old back numbers obtainable from Kraus Periodicals Co., Route 100, Millwood, NY 10546.

The Pacific Journal of Mathematics at P.O. Box 969, Carmel Valley, CA 93924 (ISSN 0030-8730) is published monthly except for July and August. Second-class postage paid at Carmel Valley, Californiä 93924, and additional mailing offices. Postmaster: send address changes to Pacific Journal of Mathematics, P.O. Box 969, Carmel Valley, CA 93924.

\section{PUBLISHED BY PACIFIC JOURNAL OF MATHEMATICS, A NON-PROFIT CORPORATION}




\section{Pacific Journal of Mathematics}

\section{Vol. 151, No. $2 \quad$ December, 1991}

Michael G. Eastwood and A. M. Pilato, On the density of twistor

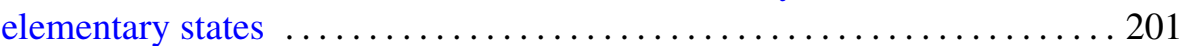

Brian E. Forrest, Arens regularity and discrete groups $\ldots \ldots \ldots \ldots \ldots \ldots 217$

Yu Li Fu, On Lipschitz stability for F.D.E ..................... 229

Douglas Austin Hensley, The largest digit in the continued fraction expansion of a rational number $\ldots \ldots \ldots \ldots \ldots \ldots \ldots \ldots \ldots \ldots \ldots . \ldots 237$

Uwe Kaiser, Link homotopy in $\mathbb{R}^{3}$ and $S^{3}$

Ronald Leslie Lipsman, The Penney-Fujiwara Plancherel formula for abelian symmetric spaces and completely solvable homogeneous

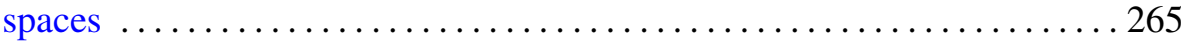

Florin G. Radulescu, Singularity of the radial subalgebra of $\mathscr{L}\left(F_{N}\right)$ and the

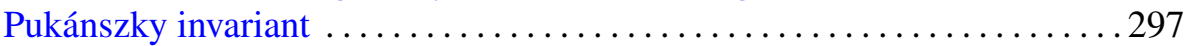

Albert Jeu-Liang Sheu, The structure of twisted SU(3) groups $\ldots . \ldots \ldots . \ldots 307$

Morwen Thistlethwaite, On the algebraic part of an alternating link . . . . . 317

Thomas (Toma) V. Tonev, Multi-tuple hulls .................. 335

Arno van den Essen, A note on Meisters and Olech's proof of the global asymptotic stability Jacobian conjecture ..................... 351

Hendrik J. van Maldeghem, A characterization of the finite Moufang hexagons by generalized homologies

Bun Wong, A note on homotopy complex surfaces with negative tangent bundles

Chung-Tao Yang, Any Blaschke manifold of the homotopy type of $\mathbf{C} P^{n}$ has the right volume 